\title{
Manifestaciones de control de padres y madres de familia en Cuenca, Ecuador, sobre sus hijos de entre 5 y 12 años
}

\author{
Manifestations of control of parents and mothers of family in Cuenca, Ecuador, \\ about their children between 5 and 12 years old
}

\begin{abstract}
Manifestações de controle de pais e mães de família em Cuenca, Equador, sobre seus filhos entre 5 e 12 anos de idade
\end{abstract}
María Dolores Palacios*a, Elsa Gardenia Conforme-Zambranob, Fernando Villavicencio $^{\mathrm{c}}$, Nube Arpid, Ruth Clavijo ${ }^{\mathrm{e}}$, Catalina Mora ${ }^{\mathrm{f}}$

\begin{abstract}
${ }^{a}$ Mst en Psicología de la Educación y Desarrollo Humano en contextos multiculturales; Universidad de Cuenca, Ecuador (ID bst Psicología de la Educación y Desarrollo Humano en contextos multiculturales. Universidad de Cuenca, Ecuador ID, ${ }^{\mathrm{c}}$ Mst Psicoterapia Familiar Universidad del Azuay, Universidad de Cuenca, Ecuador (ID) Mst Psicología de la Educación y Desarrollo Humano en contextos multiculturales. Universidad de Cuenca; Ecuador , ${ }^{\mathrm{e}} \mathrm{Mst}$ en Psicología de la Educación y Desarrollo Humano en contextos multiculturales, Universidad de Cuenca; Ecuador, ${ }^{\mathrm{f}}$ Dra.Ciencias de la Educación. Mención Investigación educativa .
\end{abstract}

Forma de citar: Dolores, M., Conforme, E., Villavicencio, F., Arpid, N., Clavijo, R. \& Mora, C. (2018). Manifestaciones de control de padres y madres de familia en Cuenca, Ecuador. Perspectivas, 3(1). 44-58

Recibido: agosto 13 de 2017

Aceptado: diciembre 03 de 2017

\section{Palabras clave}

Control parental, ausencia de control, control conductual, control psicológico, control restrictivo.

\footnotetext{
* Autor para correspondencia
} girlblue_15@hotmail.com
Resumen: En el proceso de socialización de los individuos durante su infancia, el control parental tiene múltiples manifestaciones, que obedecen a conceptualizaciones diversas. En este estudio se investiga la dimensión del control parental como a los intentos de padres y madres por regular la conducta de los hijos. Se plantea como objetivo describir las diversas manifestaciones de control como una estrategia de socialización que los padres y madres utilizan para educar a sus hijos. La investigación responde al enfoque cualitativo, es de tipo exploratorio y utiliza el análisis temático para procesar la información. Se realizaron siete entrevistas semi estructuradas a padres y madres de las familias: nucleares, reconstituidas, mono parentales paterna y materna, con niños de entre 5 y 12 años que asisten a tres escuelas de la ciudad de Cuenca. Luego del análisis de los datos, se identificaron y categorizaron cuatro tipos de control parental: ausente, conductual, psicológico y restrictivo. En conclusión, los padres y madres que participaron en esta investigación utilizan el control de forma continua en un rango que va desde la indiferencia hasta el control restrictivo. Por otra parte, se alternan las diferentes prácticas de control parental e incluso se aplica el castigo físico. Se describe a las madres de este estudio como mayormente controladoras y restrictivas con respecto a los padres: También se encontró que las madres controlan más las conductas de las hijas que las de los hijos.

https://doi.org/10.22463/25909215.1423 


\section{Keywords}

Parental control, lack of control, behavioral control, psychological control, restrictive control.

\section{Palavras chave}

Controle parental, falta de controle, controle comportamental, controle psicológico, controle restritivo

Abstract: In the process of socialization of individuals during their childhood, parental control has multiple manifestations, which obey diverse conceptualizations. In this study, the dimension of parental control is investigated, as well as the attempts of fathers and mothers to regulate the behavior of their children. The aim is to describe the various manifestations of control as a socialization strategy that fathers and mothers use to educate their children. The research responds to the qualitative approach, is exploratory in nature and uses thematic analysis to process the information. Seven semi-structured interviews were carried out with fathers and mothers of families: nuclear, reconstituted, paternal and maternal single parents, with children between 5 and 12 years old attending three schools in the city of Cuenca. After analyzing the data, four types of parental control were identified and categorized: absent, behavioral, psychological and restrictive. In conclusion, the fathers and mothers who participated in this research use control continuously in a range that goes from indifference to restrictive control. On the other hand, different parental control practices alternate and even physical punishment is applied. The mothers of this study are described as mostly controlling and restrictive with respect to the parents: It was also found that mothers control more the behaviors of the daughters than those of the children.

Resumo: No processo de socialização dos indivíduos durante a infância, o controle parental apresenta múltiplas manifestações, que obedecem a diversas conceituações. Neste estudo, a dimensão do controle parental é investigada, bem como as tentativas de pais e mães para regular o comportamento de seus filhos. O objetivo é descrever as várias manifestações de controle como uma estratégia de socialização que pais e mães usam para educar seus filhos. A pesquisa responde à abordagem qualitativa, é de natureza exploratória e utiliza análise temática para processar as informações. sete entrevistas semiestruturadas com pais de famílias foram feitas: nuclear, reconstituído, único pai e da mãe com crianças entre 5 e 12 anos freqüentando três escolas na cidade de Cuenca. Após a análise dos dados, quatro tipos de controle parental foram identificados e categorizados: ausente, comportamental, psicológico e restritivo. Em conclusão, os pais e mães que participaram desta pesquisa utilizam o controle continuamente em um intervalo que vai da indiferença ao controle restritivo. Por outro lado, diferentes práticas de controle parental são aplicadas e até mesmo a punição física é aplicada. mães deste estudo como em grande parte de controlo e restritiva no que diz respeito aos pais é descrito: Verificou-se igualmente que as mães controle sobre o comportamento das filhas dos filhos. 


\section{Introducción}

El concepto del modelo tradicional de familia (padre, madre e hijos) se ha diversificado en la actualidad para poder abarcar las diversas tipologías que existen en la realidad en relación con su estructura, jerarquía, funciones y los roles de sus miembros. Padres y madres han tenido que vivir y sobrellevar profundas trasformaciones durante las últimas décadas, lo cual ha supuesto una auténtica revolución familiar (Valdivia, 2010).

En Ecuador, las presiones económicas y las reformas políticas y sociales de los últimos tiempos han traído como una de sus consecuencias que padres y madres tengan que enfrentar problemas antes inéditos, tales como mantener relaciones afectivas cercanas con los hijos cuando están más tiempo fuera del hogar. Esta situación podría explicar estilos de crianza contradictorios en la pareja o prácticas educativas que combinan diversas estrategias (Observatorio de los Derechos de la Niñez y la Adolescencia, 2010). Así, un estudio realizado recientemente en Cuenca (Ecuador) por Palacios, Villavicencio y Mora (2015), constata que el $97,7 \%$ de los progenitores no tiene un estilo educativo definido y combina tres firmas distintas de crianza (tendencia democrática, aceptación de pautas permisivas y aceptación de determinadas estrategias autoritarias). Por otro lado, un grupo muy reducido de familias (3.3\%) mantiene un estilo educativo identificado con tendencia democrática. Estos datos coinciden con los de una investigación en España cuyos resultados señalan que el 87,2\% de los padres no tiene un estilo definido y el $12 \%$ ha elegido uno en particular, de carácter democrático (Torío, Peña e Inda, 2008; Torío, 2017).

En cuanto a las dimensiones que subyacen en los estilos educativos, el afecto ha recibido atención por parte de los investigadores en tanto que se trata de una expresión que está presente en mayor o menor medida en las prácticas de crianza. Para Fairbanks, Cassedy, Taylor, Yeates, y Wade (2013) el afecto es cercanía emocional, apoyo y cohesión, y constituye un escudo protector; sin embargo, en las prácticas de crianza también están presentes la hostilidad, la indiferencia, la negligencia e inclusive el maltrato y la violencia infantil, lo cual supone para ciertos niños y adolescentes estar en situación de riesgo en sus propias casas a manos de sus mismos padres. De acuerdo con cifras del Fondo de las Naciones Unidas para la Infancia (UNICEF, 2015), el 44\% de los menores de 5 a 17 años en el Ecuador están casi siempre expuestos a castigos violentos tales como golpes, insultos o encierro (Telégrafo, 2015). Igualmente, el Ministerio de Inclusión Económica y Social (MIES, 2017) señala que siete de cada diez niños y adolescentes en Ecuador afirmaron ser víctimas de abuso físico, sexual o negligencia familiar. Estos datos revelan que la crianza parental como una práctica de violencia es frecuente en nuestro país. Puesto que, evidentemente, constituye un elemento negativo para el desarrollo psicosocial de los hijos, debe ser denunciada y atendida hasta ser erradicada.

Ante la situación planteada se hace necesario protocolos de intervención que partan del estudio y conocimiento de lo que realmente ocurre en las familias y las prácticas de crianza en la socialización de sus hijos que emplean, de manera que padres y madres profundicen en la comprensión, por un lado de la importancia del afecto y por otro, de los efectos negativos de la violencia infantil en el desarrollo psicosocial de los niños y adolescentes. En este sentido las relaciones interpersonales constituyen uno de los rasgos esenciales en la familia vinculadas a un contexto más amplio de la comunicación y la conducta. Por otra parte, la familia se convierte en un importante escenario para el aprendizaje, pues en ella se enmarcan las estrategias y mecanismos de socialización y educación de los hijos. La función de socialización es sin lugar a dudas una de las funciones más ampliamente reconocidas de la familia, entendida en cuanto proceso mediante el cual las personas adquirimos los valores, creencias, normas y formas de conducta apropiados en la sociedad a la pertenecemos (Musitu y Cava, 2001).

Otro tema estudiado en la socialización familiar es el de las prácticas parentales. Sobre este 
particular, Hernández (2005) señala que durante la crianza y el cuidado de los niños, los padres comienzan a interactuar de diferente manera, no solamente entre ellos o con el resto de la familia, sino que establecen un espacio para los hijos en función de una nueva cotidianidad. Como es natural, las nuevas realidades y dinámicas conlleva cambios en reglas de convivencia, ajustes comunicacionales y el desarrollo de otras estrategias de afrontamiento. Para Barber y Haymon (2002), y también según Darlin y Steinberg (1993), las prácticas parentales son mecanismos que utilizan directamente los padres con miras en la socialización del niño y del adolescente, y que pueden agruparse en tres áreas: apoyo, control conductual y control psicológico.

El apoyo es una de las dimensiones que más atención ha recibido por parte de los investigadores de la socialización familiar. Por citar solo un par de ejemplos pueden revisarse al respecto los trabajos de Oliva (2006) o Rohner, Khaleque y Cournoyer (2012).

En el caso del control, que abarca las otras dos dimensiones de la socialización familiar, los investigadores no se han puesto de acuerdo sobre los aspectos que se habrían de incluir bajo dicha etiqueta (el establecimiento de límites y la aplicación de sanciones por su incumplimiento, la vigilancia o supervisión directa del comportamiento de los hijos, la exigencia de responsabilidades, el conocimiento que los padre tienen de las actividades que realizan los hijos, etc.). La mayor parte de las investigaciones no diferencian entre estas diversas dimensiones de control, por lo que resulta complejo saber cuál de ellas es la que se relaciona con el ajuste psicosocial de los hijos.

Al margen del debate académico, el control constituye ciertamente una pauta educativa usada por los padres y se considera una función parental global. Existen varios tipos de control, y sus distintos mecanismos de aplicación depende de elementos como los modelos familiares, estilos de comunicación, características de los niños, uso de la autoridad, nivel educativo de los padres e incluso las creencias culturales sobre el control parental.
Por tanto, las definiciones de control parental son diversas, pues pueden ser consideradas como límites, consistencia en las normas, uso de explicaciones o uso de castigo físico. En este sentido, Baumrind (1967) considera que el término control se refiere a los intentos de los padres para integrar al niño en de la familia y la sociedad cumpliendo con los comportamientos socialmente aceptados.

Otro conjunto de estudios indican que ciertas prácticas que enfatizan un fuerte control, obediencia $\mathrm{y}$ trabajo duro definen un estilo autoritario, que se asocia a padres estrictos y emocionalmente distantes de los hijos (Chao, 1995). Se ha sugerido que la idoneidad de estas prácticas de imposición y firmeza parental solo tendrían resultados eficaces en culturas colectivistas verticales donde las relaciones padres-hijos impliquen una relación jerárquica que se vincule con el afecto positivo y el respeto por la autoridad (Rothboum y Trommsdorff, 2007 ). En esta dirección, Schvaneveldt (2014), en una investigación con 1400 padres ecuatorianos, identificó altos niveles de control y disciplina sobre los hijos para fomentar las relaciones familiares como una práctica de la cultura colectivistahorizontal. Como se aprecia, el factor cultural predispone ciertas conductas paternas que podrían explicar las distintas manifestaciones de control con los hijos.

En otro estudio, esta vez con familias indígenas mexicanas, se revela que las mujeres perciben ser más controladas por sus madres en comparación con los hombres; sin embargo, los resultados muestran que una de cada cinco adolescentes perciben tener un nivel medio de autonomía cuando el nivel de permisividad de sus madres es medio, lo cual sugiere un balance entre autonomía y conectividad (Esteinou, 2015). En esta misma dirección, en un estudio español, Linares y Fernández (2015) sugieren que el rechazo y la disciplina rígida de la madre y la disciplina indulgente del padre, exceptuados así del control conductual, se correlacionaron de manera significativa con la agresividad impulsiva y premeditada de los hijos. 
Las investigaciones sobre el control parental, como se ha comentado, han mostrado resultados poco claros, ya que, por un lado, hay datos que apoyan la afirmación de que la supervisión como manifestación de control afecta de manera positiva la conducta de los hijos, y por otro lado, se le asocia de forma negativa con la presencia de problemas cuando se utilizan estrategias intrusivas para controlar la conducta (Betancourt, 2011).

Alarcón (2012) presenta una revisión de los trabajos que analizan el control como un concepto multidimensional que engloba distintas perspectivas de control: para Musitu, Román y Gracia (1988), el control como índice de las prácticas educativas disciplinarias parte de los presupuestos teóricos de Hoffman y diferencian el carácter disciplinario del control en disciplina inductiva o de apoyo, disciplina coercitiva y disciplina indiferente.

Por otra parte Barber y Harmon (2001) realizan una diferenciación del control según su naturaleza. Estos autores diferencian el control conductual del control psicológico. El primero se refiere a las prácticas de los padres que regulan la conducta de los niños a través de la guía y la supervisión. Estos autores describen como control psicológico aquellas conductas destinadas a interferir en los pensamientos y sentimientos de los hijos. Además, indica que afecta a varios y diversos aspectos de la personalidad de los hijos: a) interfiere en el desarrollo de la autonomía y b) pone a los menores en riesgo de sufrir baja autoestima y problemas de depresión. El control psicológico se puede manifestar en conductas parentales tales como la inducción a la culpa (que se refiere al uso de la culpa para conseguir que los menores hagan lo que los progenitores demandan); la retirada de afecto, o del afecto y el cuidado (cuando se utilizan como premio/castigo para que el menor se adecúe a los requerimientos parentales); la inducción de la ansiedad para lograr que el menor se comporte como los progenitores desean $\mathrm{y}$; finalmente, la invalidación de la perspectiva del menor, en la que se rechaza la expresión espontánea de los pensamientos, deseos y sentimientos del menor
(Barber yHarmon2002). Assory Tal (2012), por su parte, han encontrado evidencia empírica de que el control psicológico predijo la vergüenza después del fracaso. Un estudio de Baumrind, Larzelete y Owens (2010) encontró que este tipo de control es especialmente perjudicial y predictivo de problemas como la depresión, la ansiedad y la baja auto eficacia.

En las prácticas de crianza, además del control parental, está presente el castigo. Aunque es sabido que no favorece el bienestar y el desarrollo de la autonomía de los hijos, es utilizado por los padres como un mecanismo frecuente de corrección y establecimiento de comportamientos socialmente adecuados. En este sentido, Aguirre, Montoya y Reyes (2006) lo describen como el uso de la fuerza física que busca producir en el niño o niña dolor, pero no daño, con el fin de corregir y controlar su comportamiento. Sin embargo, los niños perciben el castigo como algo ambivalente y temido. En efecto, les asusta el hecho de ser castigados, y reportan que les causa dolor y les molesta (Pulido, Castro-Osorio, Peña y Ariza, 2013) .

El control parental en nuestro medio se expresa bajo formas como supervisión, acompañamiento en las tareas, limitación del tiempo, restricción de privilegios, persuasión, control de acceso a la tecnología, gritos, amenazas. Todas estas conductas son consideradas por los padres como una manera de educar a los hijos, asegurándose sobre todo de afirmar los roles de autoridad que tienen o deben tener los padres y madres.

Por la importancia de estas prácticas de control parental en el desarrollo de los hijos, el propósito de esta investigación fue describir las diversas manifestaciones de control que padres y madres familias utilizan para educar a sus hijos cuyas edades están entre los 5 y 12 años, y de esta manera profundizar en el estudio de los tipos de control parental. 


\section{Método}

Este estudio fue de tipo cualitativo, concretamente de naturaleza descriptiva, y por su intermedio se procuró explorar cómo se manifiesta el control parental y las categorías que afectan las estrategias de socialización de control que los padres y/o madres utilizan con sus hijos de entre 5 y 12 años. Estas categorías se relacionan con características asociadas a los niños, características asociadas a los padres y /o madres y los cambios actuales que afronta la familia.

\subsection{Participantes}

Los participantes fueron 6 padres y 6 madres con hijos que asisten a los distintos grados de Educación General Básica (EGB) de Cuenca, Ecuador. La selección de los participantes se realizó de acuerdo con los siguientes criterios de inclusión: 1) la tipología familiar que representa a la comunidad cuencana, 2) que tengan hijos con conductas regulares, 3 ) padres y madres que deseen participar voluntariamente en la investigación. Luego de definidos los criterios de inclusión, se reclutó a los padres de familia con la ayuda de los psicólogos de los Departamentos de Consejería Estudiantil (DECES).

La muestra fue no probabilística por conveniencia y representa la tendencia de la tipología de la familia cuencana: prevalencia de la familia nuclear, seguida de la reconstituida y en menor proporción, la familia mono parental (Palacios, Villavicencio y Mora 2015). Los participantes de la investigación tienen un nivel de instrucción básico. Las características relacionadas con el número y edad de los hijos se aprecian en la tabla 1.

Tabla 1

Características de los participantes.

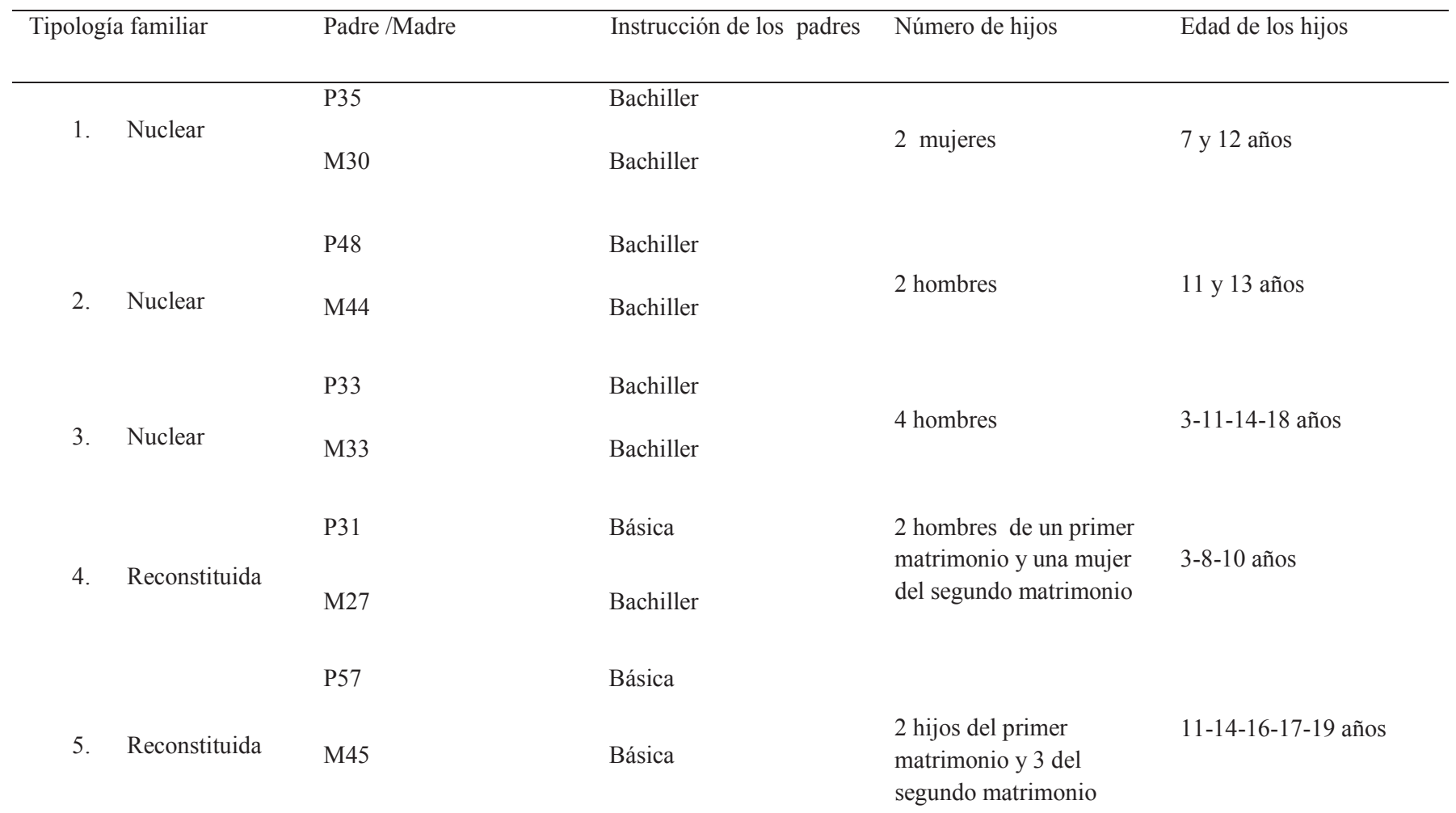



6. Monoparental
P29
Bachiller
1 hombre
7 años
paterna
7. Monoparental
M43
Básica
1 hombre
12 años

Fuente: Proceso de investigación.

\subsection{Recolección de la información}

Se diseñó una entrevista semiestructurada a partir de los siguientes temas: estructura de la familia, características asociadas al niño, características asociadas al padre/madre y los cambios que afronta la familia actual. La guía de preguntas fue analizada por dos expertos en investigación cualitativa y en socialización familiar, y validada a través de una prueba piloto (anexo 1).

Con la autorización de los directores de las escuelas, se procedió a la recolección de la información en las instituciones educativas. La duración de las entrevistas fue de aproximadamente una hora. Las intervenciones se grabaron en audio y posteriormente fueron transcritas a tiempo real. En esta investigación se tomaron en cuenta los aspectos éticos propuestos por la American Psychiatric Association (APA): 1) explicar los objetivos del estudio, 2) respetar el anonimato de los participantes,3) firma del asentimiento informado, 4) informar que los datos obtenidos tienen fines investigativos. Las entrevistas fueron realizadas por los investigadores del proyecto "Estrategias de socialización: control parental como recurso educativo de las familias cuencanas", y fueron los mismos que participaron en la elaboración de la guía y en aplicación la prueba piloto.

\subsection{Análisis de datos}

La información de las entrevistas proporcionada por los padres y madres fue procesada mediante el análisis temático Braun y Clarke (2006) de acuerdo con el siguiente procedimiento: 1) transcripción de las entrevistas,2) definición de la lista de códigos a través de una codificación de la información, con una lectura cuidadosa y un constante análisis de los relatos de los participantes 3) los códigos se ordenaron para identificarlas categorías de control parental: control indiferente, control conductual, control psicológico, y control restrictivo. Para identificar los distintos argumento de padres y madres en cada cita referenciada se utilizan las letras $\mathrm{M}$ o P para identificar si se trata del padre o la madre, seguida del número de segmento o cita asociada al conjunto de entrevistas realizadas.

Finalmente, se utilizaron diferentes estrategias para garantizar la fiabilidad de los resultados: revisión constante de las transcripciones de las entrevistas y también de los códigos. Se realizaron revisiones de los resultados y su pertinencia con los datos recolectados y los objetivos del estudio.

\section{Resultados}

El análisis de las respuestas de los participantes permitió describir las diferentes formas de control parental que los padres y madres ejercen

${ }^{1}$ Proyecto ganador del XIII Concurso Universitario de Proyectos de Investigación convocado por la Dirección de Investigación de la Universidad de Cuenca (DIUC). 
sobre la conducta de los hijos. No se encontraron contradicciones ni diferencias sustantivas entre las respuestas de los padres y las madres, de las familias nucleares o reconstituidas, sujetos de estudio de esta investigación. Por esta razón, los resultados son presentados de manera general. Los principales hallazgos de las categorías identificadas responden a los planteamientos de por Rohner (2012), quien señala que el control parental constituye una práctica continua que va de la permisividad al control restrictivo. Sin embargo, del análisis de los resultados emergieron dos categorías que se ubicaron entre estas dos prácticas ubicadas en los polos extremos (ver tabla 2).

Tabla 2

Códigos y dimensiones de las categorías de control parental.

\begin{tabular}{ccc}
\hline Códigos & Dimensiones & Categorías \\
\hline Ausencia de normas & Control indiferente & \\
Flexibilidad & & \\
\hline Supervisión & Control conductual & \\
Implicación materna y paterna & \\
Disciplina & \\
Manipulación & Control psicológico \\
Insultos y gritos & \\
\hline Restricción de privilegios & Control restrictivo \\
Castigo & \\
\hline
\end{tabular}

Fuente: Proceso de investigación.

A continuación se detallan los principales hallazgos en cada una de las dimensiones identificadas.

\subsection{Control indiferente (ausencia de control)}

Al preguntarles: ¿cómo establecen las normas a los hijos? y ¿qué hacen para qué sus hijos las cumplan?, las opiniones de los participantes sobre la crianza expresaron pasividad y flexibilidad ante el incumplimiento de las normas de sus hijos. Esto evidencia que en los intentos de los padres por educar bien a los hijos, algunos pueden resultar poco exigentes para en el cumplimiento de las mismas.
De los relatos de los padres emergen las siguientes manifestaciones de control indiferente:

\subsubsection{Ausencia de normas}

Las respuestas frente a cómo poner normas a los hijos fueron diversas; sin embargo, algunos padres y madres de familia entrevistadas expresaron que no es fácil, tanto establecer normas como hacer que los hijos las obedezcan. Señalaron que es difícil porque no quieren que sus hijos tengan la misma experiencia de maltrato que recibieron de sus padres; otros señalan que vienen cansados del trabajo, que no saben cómo poner reglas, que ahora con los derechos de los niños no se les puede 
hablar duro, ni llamarles la atención. Se presentan las respuestas más representativas de esta conducta paterna caracterizada por la ausencia de normas:

Entonces para mí fue duro ponerle normas porque yo a mi hijo... yo soy de las personas que no me gusta que derrame una lágrima, pero a veces es necesario (P 29).

Me pongo hacer yo mismo lo que yo les mando (M30).

Estas prácticas paternas de control indiferente carecen de firmeza y coherencia para controlar la conducta de los hijos, presentan niveles mínimos de control, de lo que se deduce que este tipo de padres no responde de ninguna manera ante el incumplimiento de normas. De acuerdo con Parra (2005), el control indiferente parece relacionarse con el nivel educativo de los padres, pues el autor ha encontrado que los estilos indiferentes son más frecuentes entre los progenitores sin estudios o con niveles bajos. Esos resultados encuentran similitud con los de este estudio, pues los padres de la muestra analizada tienen un nivel medio de bachillerato.

\subsubsection{Flexibilidad}

Un hallazgo importante que se evidencia de los relatos de los padres y madres tiene que ver con el hecho de que cuando son flexibles en el establecimiento de las reglas para el comportamiento de los hijos, estas se cumplen parcialmente o no se cumplen. Se considera, que esta flexibilidad podría estar relacionada con las características de los hijos: edad, orden de nacimiento, condición de salud del niño o comportamiento.

Bueno, a veces sí. Hay veces cuando está enfermito no le digo nada. O sea, le dejo que esté en la tele, que esté acostadito... Pero ya en los días normales hay veces que sí me gana. (M30).

“¡Hijo ven a ayudarme acá o pásame el escamel!” [Herramienta para labrar]. Él decía: “¡Ayi lo siento, estoy ocupado, estoy cansado...". Le decía: "¡Muévete, ayúdame, mmm....". Entonces le decía: “¡Mijo [hijo], muévete!”, le rogaba. Me decía: “„No!”. Entonces le decía: "Ya, ya, ya...". (P 29).

De las expresiones de los padres se deduce que cuando los niveles de control son escasos y altos niveles de afecto, hablamos de padres consentidores y cuando la ausencia de control es extrema, acompañada de afecto, hablamos de padres permisivos.

Los testimonios anteriormente citados, sin embargo, se vinculan más con un talante caracterizado por Rodríguez, del Barrio y Carrasco (2010) como un estilo consentidor permisivo, al que tiende gran parte de los padres actuales, que consiste básicamente en un estilo mix to que presenta las características del democrático (respecto al afecto y preocupación, que incluso conduciría a la sobreprotección de los hijos), pero al mismo tiempo participa de un estilo negligente, ya que la permisividad es alta.

\subsection{Control conductual}

En la socialización familiar, las prácticas paternas buscan regular el comportamiento de los hijos para que aprendan que en las interacciones sociales, el cumplimiento de las normas ayuda a mantener el orden para que sean sujetos competentes y autónomos. De las opiniones recogidas de los padres y madres de este estudio, se pueden apreciar frases que manifiestan conductas de supervisión, monitoreo, implicación materna/ paterna $\mathrm{y}$ disciplina:

\subsubsection{Supervisión}

Se entiende, la supervisión o el control como el conocimiento que los padres y madres tienen de las actividades de los hijos. Desde esta perspectiva se resalta la respuesta de algunos participantes que dan valor al seguimiento o acompañamiento que 
deben hacer los padres a las actividades diarias de sus hijos:

Siempre estamos atrás, no le damos mucho tiempo. Si ella no está en la casa nosotros estamos atrás de ella, le llamamos. (M.30).

"! Mami!-dicen-, "Tengo que ir a hacer un trabajo". Digo: "Está bien. Anda donde tu amigo, lo que sea... Te vas y estoy al pendiente. Pero ven pronto". (M.43).

Le pregunto todo el tiempo, si ya hizo los deberes, si estudiaste, si tiene pruebas.... (M.45)

Llaman la atención en los relatos que sean las madres quienes ejercen más control sobre la conducta de los hijos. Estos datos son similares a los encontrados por Esteinou (2015), que indicaba que las hijas perciben a las madres más controladoras que a los padres. Asimismo, García Linares y Fernández (2015), en una investigación realizada en España, muestran que la disciplina rígida es ejercida por la madre frente a la falta de disciplina y la indulgencia del padre. Finalmente, de las narrativas de las madres se deduce que la supervisión la monitorización es una manifestación de control activo que busca regular la conducta de los hijos.

\subsubsection{Implicación materna y paterna}

Se trata de una práctica de control más indirecta sobre la conducta de los hijos, caracterizada por mostrar afecto y mostrarse empáticos con ellos (Musito et al. 1988). Los padres y madres permiten a los hijos que realicen tareas, pero buscan estar siempre presentes física o afectivamente detrás de los hijos. Así lo recogen las siguientes citas:

O sea, todos los días conversamos. Y... "Mijo, ¿cómo te fue en el colegio? ¿Qué tienes? ¿Qué notas sacaste? ¿Qué te pusieron en el deber?". O sea, en ese sentido tenemos buena comunicación con mis hijos... (M. 44).
Yo siempre estoy pendiente de las dos hijas, en llevar y traer a las hijas de la escuela.(P.33).

Estas respuestas manifiestan que una forma de control conductual en la socialización familiar se expresa a través de la presencia física y de las conductas de apoyo y del afecto que se brinda a los hijos.

\subsubsection{Disciplina}

La disciplina como manifestación de control conductual puede ser inductiva, es decir, conducir al ejercicio positivo de la paternidad en tanto que se basa en el diálogo, la reflexión y promueve el desarrollo de la autonomía en los hijos (Musito et al. 1988). Los relatos que apoyan esta manifestación son los siguientes:

No dejo que digan malas palabras, no hago que se porten mal, pero jamás he llegado a la agresión física. Solo palabras firmes.(M.33).

O sea, a ella le hacen más caso porque... o sea, ¿cómo le digo?, ella es más seria. $\mathrm{O}$ sea... no es golpes, no es gritos, pero es más seria. Ella ordena y ellos hacen caso (P.57).

Con base en estas citas de los padres y madres puede afirmarse que el control es una estrategia disciplinaria que está relacionada con un tipo de vigilancia firme de los padres hacia las actividades de los hijos, que busca regular, monitorear y supervisar el cumplimiento de las normas y reglas establecidas. Se considera que las prácticas de control conductual manifestadas en la supervisión, implicación afectiva y disciplina sobre los hijos en la cultura cuencana, se relaciona con los postulados de Rohner y Carrasco (2014), quienes señalan que la influencia cultural mantiene las conductas parentales.

\subsection{Control psicológico}

Los participantes del estudio reconocieron que para establecer reglas y normas sobre los hijos muchas 
veces emplean gritos, críticas e ironías, además de amenazas de castigo. Para Barber y Harmon (2002) estas conductas son intrusivas porque manipulan tanto pensamientos como sentimientos de los hijos. A continuación, se analizan las manifestaciones de control psicológico que emergieron del análisis de los relatos de los padres y madres.

\subsubsection{Manipulación}

Las opiniones de los padres y madres frente a las formas de establecer reglas fueron diversas; sin embargo, algunos participantes indicaron que muchas veces la relación con los hijos se vuelve conflictiva al momento de poner reglas y que algunas veces incurren en los reproches, la victimización y en mostrar el sacrificio que hacen para educar a los hijos:

Y digo: "Mejor. ¿Sabes qué? Voy a dejarte donde tu papá, porque a mí ni hablándote duro haces caso. Allá no vas a morir de hambre. Tu papá tiene mucho para mantenerte". (M.43).

Estos relatos describen conductas de manipulación, entendidas como prácticas parentales que buscar dominar al hijo en interés de las demandas o expectativas de los padres y madres mediante el manejo de la culpa y la ansiedad como mecanismos para conseguir que los hijos hagan lo que los padres dicen.

Yo simplemente le digo: "Mijo [hijo]. Está bien. No quieres estudiar y tú sabes que yo hago lo mejor por ti. Me mato trabajando por ti para verte bien, que estudies...Pero si no quieres, mi amor, está bien” (P.33).

Esta última cita recoge otra forma de manipulación, que consiste en la retirada de afecto, amor, cuidado y del apoyo que los padres brindan a sus hijos. Esta manipulación busca generar culpa para lograr que los hijos hagan lo que los padres quieren (Barber y Harmon 2002).

\subsubsection{Insultos y gritos}

Otra forma de control psicológico que los padres y madres emplean para controlar la conducta de los hijos son los insultos, gritos y amenazas. Así lo expresan los siguientes testimonios.

¡Solo en la computadora, longos vagos! [Muchachos ociosos]. Ahí están echados [acostados] en la cama! ¡Parecen puercos! (M.33).

Le hablo, le grito duro las cosas, le digo las cosas cuando no hace los deberes, por más que le digo no obedece. Él (el hijo) dice:"Mañana... mañana... pero no mismo". Y es ahí que le grito.(M43).

No me gusta pegarles ni golpearles pero si tengo una voz medio gruesa y digo:" ¡No hagas eso!". Aunque al escuchar se ponen bravos [molestos] (M.45).

Se considera que las prácticas que utilizan los padres y madres entrevistados son conductas posesivas, controladoras y alienantes en el desarrollo saludable de los hijos, y que podrían ser predictivas tanto de los problemas externalizados, como consumo de alcohol o tabaco y la conducta agresiva, como de conductas internalizadas, como la depresión (Betancour y Andrade, 2011 ). Estos hallazgos concuerdan con investigaciones que prueban que los hijos que perciben críticas excesivas, chantaje e inducción a la culpa presentan más problemas de conductas (Olivia, 2006; Reitz, Dekovic y Meijer 2006; Soenens y Vansteenkiste $2010)$.

\subsection{Control restrictivo}

El control restrictivo, como lo señalan Musitu, et al. (1998), se refiere a las técnicas disciplinarias que utilizan la aplicación de la fuerza y del poder de los progenitores, incluyendo la amenaza, privación de privilegios y afectos, y la restricción de los mismos. Para indagar la forma en que los 
padres regulan la conducta de los hijos se realizaron preguntas que pretendían conocer qué hacen para que los hijos les obedezcan.

\subsubsection{Restricción de privilegios}

Estas prácticas son intentos de los padres para controlar el comportamiento de los hijos, establecer normas sociales, escolares, regular las emociones o modificar sus comportamientos inadecuados; sin embargo, estas conductas paternas como estrategias de socialización afectan el desarrollo de la competencia social e inhibe la autonomía de los hijos (Soenens y Vansteenkiste, 2010). Las expresiones de este tipo de control fueron identificadas en los relatos de padres y madres momentos de la entrevista como los que se presentan a continuación:

Yo le quité el teléfono. Le dije que tenía un horario para utilizarlo, por ejemplo, media o una hora. $\mathrm{O}$ cuando pasaba en la casa tenía el celular hasta las 7 u 8 de la noche (P 33).

Le quité la computadora. Desde que empezó el año empezó con sus amiguitos, que le dieron grabando juegos. Entonces venía del colegio a los juegos: “¡Mami, solo una media hora...!”. Pero se pasaba horas en el juego (M44).

Les digo: "Mijos [hijos], tienen que hacer ayudar en las tareas de la casa o si no, no hay televisión o algún programa”. (P 57).

Los padres y madres manifiestan que al restringir el uso de estos recursos tecnológicos y al limitar las horas para el uso del internet, lo hacen más para obtener una conducta deseada que por educarles sobre el potencial riesgo que implica el mal uso de la tecnología y las redes sociales.

\subsubsection{Castigo}

Otra práctica de control parental evidenciada en los relatos de los padres es el castigo. Varios participantes respondieron que cuando los hijos no obedecen utilizan golpes para controlar su conducta.
Este patrón de comportamiento podría explicar las expresiones de castigo recogidas en los relatos de los participantes de este estudio:

A veces [mi hijo] cuenta cuando le he dado sus buenos... o sea, le he dado duro [golpes]. No puedo darle todos los días ni tampoco todas las semanas. Tengo que pensar qué es lo que le duele para darle un castigo (M45).

Con una soga que sabe estar ahí o una correa o el zapato... Pero evito el zapato. Le doy con la correa o la soga (M33).

Estas conductas son similares a las encontradas en un estudio realizado en Colombia por Pulido, Castro-Osorio, Peña y Ariza, (2013 ) que reportó prácticas de castigo como golpes, fuetazos, cachetadas y palmadas a los hijos e hijas, y también bañarles en agua fría. Estas prácticas están justificadas por la creencia de que si no se aplica el castigo los hijos no aprenden. Por eso no se consideran las consecuencias negativas que en los niños tiene el castigo a nivel psicológico, físico, social y que se asocia con la baja autoestima y percepción negativa de sí mismo, así como con comportamientos antisociales en la infancia y adolescencia (Aguirre, Montoya y Reyes 2006; Buitrago-Peña, GuevaraJiménez y Cabrera-Cifuentes, 2009).

\section{Conclusiones}

Los resultados de este estudio muestran que padres y madres de familia manifiestan diferentes formas de control parental para educar y controlar la conducta de los hijos. De los relatos de los participantes se deduce que en la crianza de los hijos se manifiesta el control de una forma continua, desde un control indiferente (inexistente) hasta un control restrictivo. Dicho de otro modo, los padres y madres alternan prácticas de control indiferente, conductual, psicológico, restrictivo. Estas manifestaciones continuas del control parental podrían están relacionadas con las características 
de los hijos en cuanto a su edad y características de salud.

Sobre el control conductual, se estima que los padres y madres sujeto de estudio ejercen una conducta activa mediante la supervisión y monitoreo para conocer lo que hacen sus hijos y de esta manera poder regular y controlar su conducta. De la misma manera se puede concluir que en la crianza de los hijos está presente un control psicológico a través del cual los padres se inmiscuye en la vida de sus vástagos, principalmente mediante conductas de manipulación, inducción a la culpa y amenazas para ejercen una presión psicológica sobre las emociones, sentimientos y conducta de los hijos.

Además, los resultados permiten conocer que en cuanto al control restrictivo, los padres y madres de este estudio emplean el castigo tanto para modificar la conducta como para establecer comportamientos esperados para los hijos. Se considera que la influencia sociocultural del contexto cuencano podría ser un factor que explicaría el mantenimiento de esta práctica coercitiva.

Los resultados de este estudio, finalmente, aportan importantes información que permite poner en evidencia que los padres y madres desconocen cómo establecer reglas sobre sus hijos, cómo regular la conducta y ejercer un control respetuoso a través del afecto, el involucramiento y la inducción positiva, de manera que los hijos alcancen mayores niveles de bienestar, autonomía y competencias psicosociales. Por lo expuesto, es importante la implementación de programas de educación parental positiva que favorezcan prácticas parentales más asertivas.

\section{Referencias}

Aguirre, A., Montoya, L. y Reyes, J. (2006). Crianza y castigo físico. Diálogos y discusiones en la psicología contemporánea, 1, 20-25.

Alarcón, A. (2012). Estilos parentales de socialización y ajuste psicosocial de los adolescentes: Un análisis de las influencias contextuales en el proceso de socialización.
Valencia: Universidad de Valencia.

Assor, A., \& Tal, K. (2012). When parent's affection depends on child's achievement: Parental conditional positive regard, self-aggrandizement, and shame and coping in adolescents. Journal of Adolescence, 35, 249-260.

Barber, B. K., \& Harmon, E. L. (2002). Violating the self: Parental psychological control of children and adolescents. In B. K. Barber, (Ed.), Intrusive parenting: How Psychological Control Affects Children and Adolescents (pp. 15-52). Washington: American Psychological Association.

Baumrind, D., 1967. Child care practices anteceding three patterns of preschool behavior. Child Development, 37(4), 887-907.

Baumrind, D., Larzelere, R. E., \& Owens, E. (2010). Effects of preschool parents' power assertive patterns and practices on adolescent development. Parenting: Science and Practice, 10(3), 157-201

Betancourt, D., Andrade, P. (2011). Control parental y problemas emocionales y de conducta en adolescentes. Revistacolombiana de psicología,20, 27-41.

Belsky, J. (2005). Family influences on psychological development. Psychiatry, 4(7), 41-44. doi: 10.1016 / j.mppsy.2008.05.006

Braun, V. \&Clarke, V. (2006). Using thematic analysis in psychology, Qualitative Research in Psychology, 3:2,77-101. http://dx.doi. org/10.1191/1478088706qp0630a .

Buitrago-Peña, M., Guevara-Jiménez, M. y CabreraCifuentes, K. (2009) Las representaciones sociales de género y castigo y su incidencia en la corrección de los hijos. Educación y educadores, 12(3), 53-71.

Chao, R.K., 1995. Beyond parental control and authoritarian parenting style: Understanding Chinese parenting through the cultural notion of training. Child Development, 65(1), 111-119.

Darling, N. \& Steinberg, L. (1993) Parenting style as context: an integrative model.Psychological Bulletin, 114, 487-796. 
El Telégrafo. (05 de marzo de 2016). El 44\% de los menores de 5 a 17 años está expuesto a castigos violentos. Recuperado de http://www. eltelegrafo.com

Esteinou, R. (2015). Autonomía adolescente y apoyo y control parental en familias indígenas mexicanas.Revista latinoamericana de ciencias sociales, 13, 749-766. doi:10.11600/169271 $5 x .13214230114$.

Fairbanks, J., Cassedy, A., Taylor, H., Yeates, K., \& Wade, S. (2013). Fairbanks. Rehabilitation Psychology, 58, 223-232.

Hernández, A. (2005). Familia, ciclo vital y psicoterapia sistémica breve. Bogotá: Editorial El Buho.

Linares, M. C. G., y Fernández, M. D. L. V. C. (2015). Las prácticas educativas paternas y la agresividad premeditada e impulsiva de los hijos adolescentes. Psicología conductual, 23(1), 161179.

Martínez, I., García, J.F. y Yubero, S. (2007). Parenting styles and adolescents'self-esteem in Brazil.PsychologicalReports, 100, 731-745.

Ministerio de Inclusión Económica y Social (MIES). (2017). Perfil sociodemográfico de la población infantil en el Ecuador. Boletín InfoInclusión. http://info.inclusion.gob.ec/infomies/descargas documentos/SISTEMA_DE_INFORMACION/ S I S T EM A D E I N F O R M A C I O N ESTADISTICA/INFO_INCLUSION_02.pdf

Musitu, G., M.J. Cava, 2001. La familia y la educación (1a ed.). Barcelona: Octaedro.

Musitu, G., Roman, J., y Gracia, E. (1988). Familia y educación. Prácticas educativas de los padres en la socialización de los hijos. Barcelona: Labor Universitaria.

Observatorio de los Derechos de la Niñez y la Adolescencia, 2010. Los niños y las niñas del Ecuador a inicios del siglo XXI. Quito: Noción.

Oliva Delgado, A. (2006). Relaciones familiares y desarrollo adolescente. Anuario de psicología, 37(3), 209-223.

Palacios, M., Villavicencio, F. y Mora,C. (2015). Evaluación de los estilos educativos familiares en la ciudad de Cuenca. Maskana, 6(2), 31-45.

Parra, A. (2005). Familia y desarrollo adolescente: un estudio longitudinal sobre trayectorias evolutivas. Tesis Doctoral no publicada. Sevilla: Universidad de Sevilla.

Penalva, C.; Alaminos, A.;Frances, F. ySantacreu, O. (2015). La investigación cualitativa: técnicas de investigación y análisis con Atlas ti. PYDLOS Ediciones.

Pulido, S., Castro-Osorio, J., Peña, M., y ArizaRamírez, D. (2013). Pautas, creencias y prácticas de crianza relacionadas con el castigo y su transmisión generacional. Revista Latinoamericana de ciencias sociales, niñez y juventud, 11(1), 245-259.

Reitz, E., Dekovic, M. \& Meijer, A. M. (2006). Relations between parenting and externalizing and internalizing problem behavior in early adolescence: Child behaviour as moderator and predictor. Journal of Adolescence, 29, 419-436.

Rodríguez, M., Del Barrio, V. y Carrasco, M. (2010). El libro de la Familia. Un G.P.S. Educativo. Disponible en http://www.defensor-and.es defensor@defensor-and.es.

Rodrigo, M., y Palacios, J. (1998). Familia y desarrollo humano. Madrid: Alianza Editorial.

Rohner, R. P., Khaleque, A., y Cournoyer, D. E. (2012). Introduction to parental acceptancerejection theory, methods, evidence, and implications.Journal of Family Theory \& Review, 2, 73-87.

Rohner, R., y Carrasco, M. Á. (2014). Teoría de la aceptación-rechazo interpersonal (IPARTheory): Bases conceptuales, método y evidencia empírica [Interpersonal Acceptance-Rejection Theory (IPARTheory): Theoretical Bases, Method and Empirical Evidence ] Antecedentes. Acción Psicológica, 11, 9-26.

Rothbaum, F., \& Trommsdorff, G. (2007). Do roots and wings complement or oppose oneanother?: The socialization of relatedness and autonomy in cultural context. In J. E. Grusec, \& P. D. Hastings (Eds.).Handbook of socialization: 
Theory and research (pp.461-489). New York, NY: Guilford Press.

Soenens, B. \& Vansteenkiste, M. (2010). A theoretical upgrade of the concept of parental psychological control: proposing new insights on the basis of self-determination theory. Developmental Review, 30, 74-99.

Schvaneveldt, P. L. (2014). Parenting in Ecuador: Behaviors that promote social competence. In $\mathrm{H}$. Selin (Eds.), Parenting Across Cultures (pp. 323334). Netherlands: Springer. doi: 10.1007/97894-007-7503-9_24

Torio, S. (2017). ¿Cómo Educar? ¿Lo estamos haciendo bien? Contribuyendo al actual debate de la literatura acerca del estilo educativo parental óptimo. Pedagogía social. Revista interuniversitaria, 29, 9-18. doi: 10. SE7179/ PSRI_2016.29.00

Torio, S., Peña, V., e Inda, M. (2008). Estilos de educación Ffmiliar. Psicothema, 20(1), 62-70. Recuperado de http://www.redalyc.org/articulo. oa? id $=72720110$.

Valdivia, M. del C. (2010). Madres y padres competentes. Claves para optimizar las relaciones con nuestros hijos e hijas. Barcelona: Grao. 